\title{
Análise espacial do índice de desenvolvimento humano municipal na região semiárida brasileira
}

\author{
Spatial Analysis of Human Development Municipal Index in the Brazilian Semiarid Region
}

\author{
Hélder Gramacho dos Santos ${ }^{1}$ \\ José Antonio Moura e Silva ${ }^{1}$ \\ José Luiz Portugal ${ }^{2}$
}

\author{
${ }^{1}$ Instituto Nacional de Colonização e Reforma Agrária - INCRA \\ Superintendências Regionais do Estado da Bahia (SR-05) e do Médio São Francisco (SR-29) \\ Av. Ulisses Guimarães, 640 - $1^{\circ}$ Andar - Centro Administrativo - Salvador, BA - CEP: 41213-000, Brasil. \\ agrohelder@gmail.com; mourajantonio@yahoo.com.br \\ ${ }^{2}$ Universidade Federal de Pernambuco-UFPE \\ Centro de Tecnologia e Geociências / Departamento de Engenharia Cartográfica \\ Av. Acadêmico Hélio Ramos, s/n - $2^{\circ}$ andar - Cidade Universitária - Recife, PE - CEP: 50740-530, Brasil. \\ joseluiz.portugal@gmail.com
}

Artigo - Submissão: 07/07/2015 - Aceito em: 14/12/2015

\begin{abstract}
RESUMO - A região Semiárida Brasileira é um espaço geográfico com área de $980.133,079 \mathrm{~km}^{2}$, caracterizada por uma precipitação média anual inferior a 800 milímetros, índice de aridez de até 0,5 e risco de seca maior que $60 \%$. Abrange 9 estados, 1135 municípios e sua população é de 22.598 .318 habitantes. O Índice de Desenvolvimento Humano dos Municípios (IDHM) é um indicador que permite conhecer as oportunidades de crescimento e de escolhas que estão disponíveis no nível municipal para que as pessoas tenham a vida que desejam. O Atlas do Desenvolvimento Humano no Brasil 2013 disponibiliza o IDHM e suas três componentes: IDHM Renda, IDHM Educação e IDHM Longevidade, calculados a partir de dados do Censo Demográfico do Brasil do ano de 2010. O objetivo do trabalho foi descrever e visualizar distribuições espaciais, descobrir padrões de associação, identificar aglomerados e situações atípicas nos dados do IDHM da região Semiárida Brasileira através da Análise Exploratória de Dados Espaciais (AEDE). Os resultados mostram a existência de autocorrelação espacial no IDHM. Foram identificados clusters de altos e baixos valores com diferentes padrões de agregação. Tais resultados podem provocar a realização de estudos mais aprofundados para entender os fatores de agregação, bem como sugerir regiões prioritárias para atuação por parte dos governos estaduais.
\end{abstract}

Palavras-chave: autocorrelação espacial, índice de Moran, análise exploratória de dados espaciais.

\begin{abstract}
Brazilian semiarid region is a geographic area with $980.133,079 \mathrm{~km}^{2}$, characterized by an average annual rainfall less than $800 \mathrm{~mm}$, aridity index up 0,5 and dry risk of greater than $60 \%$. Covers 9 states, 1135 counties and a population of 22.598.318 inhabitants. Human Development of Municipalities Index (IDHM) allows to know the reality of human development of the municipalities. Human Development Brazil Atlas in 2013 offers the IDHM and its three components: IDHM Income, IDHM Education and IDHM Longevity, calculated from Census data from Brazil in 2010. Exploratory Spatial Data Analysis (ESDA) was used in order to describe and visualize spatial distributions, discover patterns of association, to identify clusters and atypical situations IDHM on data from the Brazilian semiarid region. Results show that there are spatial autocorrelation in IDHM. Clusters of high and low values were identified with different patterns of aggregation. These results may lead to conduct further studies to understand the factors of aggregation and suggest priority areas for action by state governments.
\end{abstract}

Keywords: spatial autocorrelation, Moran index, exploratory spatial data analysis.

\section{INTRODUÇÃO}

Desenvolvimento humano é o processo de ampliação das liberdades das pessoas, no que tange a suas capacidades e as oportunidades a seu dispor, para que elas possam escolher a vida que desejam ter. A medida deste desenvolvimento é dada pelo Índice de Desenvolvimento Humano IDH (PNUD, 2013).
O Índice de Desenvolvimento Humano dos Municípios (IDHM) é uma adaptação cuja metodologia de cálculo foi construída para melhor se adequar à realidade brasileira, adaptando-se às bases de dados do Censo Demográfico Brasileiro e às características inatas aos municípios. Esse indicador pode ser aplicado para diversas unidades espaciais de análise, como países, estados, municípios entre outras. 
De acordo com PNUD (2013) o IDHM é um importante indicador devido a três fatores: o primeiro é ampliar a visão de que o desenvolvimento se limita a crescimento econômico para o conceito de desenvolvimento centrado nas pessoas; o segundo é que ao sintetizar uma realidade complexa em um único número, o IDHM e suas três componentes (renda, educação e longevidade) viabilizam a comparação entre os municípios Brasileiros ao longo do tempo e o terceiro é que o ranking do IDHM estimula formuladores e implementadores de políticas públicas no nível municipal a priorizar a melhoria da vida das pessoas em suas ações e decisões.

Em que pese no ano de 2013 o Brasil tenha sido considerado pelo Relatório de Desenvolvimento Humano do Programa das Nações Unidas para o Desenvolvimento como um país de "alto desempenho", internamente o país é marcado por grandes desigualdades, principalmente quando comparamos os municípios localizados no sul e sudeste do país com os localizados na região nordeste, agravando-se ainda mais a situação quando olhamos para os municípios do nordeste localizados na região Semiárida.

A região Semiárida Brasileira é um espaço geográfico com área de 980.133,079 km², caracterizada por uma precipitação média anual inferior a 800 milímetros, índice de aridez de até 0,5 ou ainda risco de seca maior que $60 \%$. Abrange nove estados, 1135 municípios e sua população é de 22.598.318 habitantes o que corresponde a cerca de $11,85 \%$ da população Brasileira (INSA, 2012).

Conhecer esta realidade é fundamental para a tomada de decisão no que se refere à implantação de programas governamentais e também para aperfeiçoamento dos programas existentes que tenham como objetivo melhorar as condições de vida desta população.

A técnica da análise exploratória de dados espaciais tem como objetivos descrever a distribuição espacial, os padrões de associação espacial (clusters espaciais), verificar a existência de diferentes regimes espaciais, outras formas de instabilidade espacial (nãoestacionariedade) bem como identificar observações atípicas (outliers) (Perobelli, Faria e Ferreira, 2006).

O objetivo do trabalho foi analisar os resultados da aplicação da técnica de Análise Exploratória de Dados Espaciais (AEDE) nos dados do IDHM dos 1135 municípios que fazem parte da região Semiárida Brasileira.

\subsection{Análise Exploratória Espacial}

A utilização de métodos convencionais segundo Gonçalves (2007), referindo-se às regressões múltiplas e à análise visual de mapas, não são confiáveis para detectar agrupamentos e padrões espaciais significativos sendo portanto inadequadas para análise de dados georreferenciados. Uma alternativa para solucionar estas dificuldades é a AEDE.
O primeiro passo para a realização da AEDE é a obtenção da matriz da proximidade espacial (W), do vetor dos desvios $(\mathrm{Z})$ e do vetor de médias ponderadas (Wz). A matriz de proximidade espacial é uma ferramenta geral e útil para descrever o arranjo espacial dos objetos (Neves et al, 2000). W é uma matriz quadrada, com $n^{2}$ elementos, onde cada elemento, $\mathrm{w}_{\mathrm{ij}}$, representa uma medida de proximidade espacial entre o polígono $\mathrm{i}$ e o polígono $\mathrm{j}$, sendo n, o número total de objetos. Geralmente utiliza-se como medida de proximidade:

objetos com fronteira comum, $\mathrm{w}_{\mathrm{ij}}=1$;

objetos sem fronteira comum, $\mathrm{w}_{\mathrm{ij}}=0$.

A construção da matriz de proximidade espacial é a primeira etapa para quem deseja realizar a AEDE. Os programas constroem a matriz de proximidade espacial utilizando informações da topologia, extraída da representação gráfica dos objetos. Ela é construída automaticamente após a definição da estratégia de construção e do peso que cada vizinho teria.

O próximo passo é definir o atributo para que seja possível calcular o vetor de desvios Z. Na sequência é calculada a média $(\mu)$ dos valores dos atributos, considerando os $\mathrm{n}$ objetos. Cada elemento $\mathrm{i}$ de $\mathrm{Z}, \mathrm{Z}_{\mathrm{i}}$, é obtido subtraindo-se o valor da média, do valor do atributo correspondente $\left(z_{i}=y_{i}-\mu\right)$. Para este cálculo são utilizados os valores contidos na tabela de dados do atributo selecionado.

O terceiro elemento básico, o vetor de médias ponderadas $(\mathrm{Wz})$, também é calculado automaticamente por meio da multiplicação matricial do vetor transposto dos desvios, pela matriz de proximidade espacial com linhas normalizadas, onde cada elemento de uma linha $\mathrm{i}$ qualquer, originariamente com valor 1, é dividido pelo número de elementos não nulos da mesma linha. Desta forma, como resultado, cada elemento $\mathrm{wz}_{\mathrm{i}}$, contém um valor correspondente à média dos desvios dos vizinhos ao objeto i (Neves et al, 2000).

A partir destes elementos são calculados o Índice de Moran Global e o Indicador Local de Associação Espacial (LISA), bem como é gerado o diagrama de espalhamento de Moran os quais podem ser visualizados num SIG.

\section{2 Índice Global de Associação Espacial: Índice de Moran I}

A expressão matemática do Índice Global de Moran, I, a partir dos elementos descritos anteriormente é dada pela Equação (1):

$$
I=\frac{Z^{t} \cdot W_{z}}{Z^{t} \cdot Z}
$$

Eq. 1

Onde:

$\mathrm{Z}^{\mathrm{t}}$ é o vetor dos desvios transposto;

$\mathrm{W}_{\mathrm{z}}$ é o vetor de médias ponderadas;

$\mathrm{Z}$ é o vetor dos desvios.

O índice global de Moran varia entre -1 e 1 . A sua interpretação prática é: valores positivos indicam que existe uma associação espacial (autocorrelação espacial) positiva para a variável observada. Quanto mais próximo for o índice global da unidade, mais forte é a dependência 
espacial entre as observações. De forma inversa, se o índice assumir valores negativos, isto indica que existe, então, uma autocorrelação espacial negativa entre as observações. Valores próximos de zero indicam que existe pouca dependência espacial entre as observações NEVES e LUIZ (2006).

\section{3 Índice Local de associação espacial (LISA)}

Uma vez que o índice Global de Moran analisou de maneira geral o conjunto dos dados e identificou a existência da autocorrelação espacial, é possível então analisar cada elemento do conjunto em nível local, buscando a identificação de aglomerados de elementos que apresentem para os valores dos seus atributos dados semelhantes os quais são chamados de "clusters". Os elementos que apresentam comportamento atípico com relação aos seus vizinhos são chamados de "outliers", bem como regimes espacias diferenciados.

ANSELIN, (1995) sugere que de forma geral um Indicador Local de Associação Espacial (LISA) deve atender aos seguintes requisitos:

1. o LISA para cada observação deve fornecer uma indicação da extensão da aglomeração espacial significativa de valores similares em torno dessa observação;

2. a soma de todas as observações do LISA seja proporcional ao indicador global de associação espacial.

Neste trabalho foi utilizado como LISA o Índice Local de Moran que pode ser obtido por meio da Equação (2):

$$
I_{i}=\frac{Z_{i} \cdot W z_{i}}{\sigma^{2}}
$$

onde:

$\mathrm{I}_{\mathrm{i}}$ : índice local para o objeto $\mathrm{i}$;

$\mathrm{Z}_{\mathrm{i}}$ : valor do desvio do objeto $\mathrm{i}$;

$\mathrm{Wz}_{\mathrm{i}}$ : valor médio dos desvios dos objetos vizinhos de $\mathrm{i}$; $\sigma^{2}$ : variância da distribuição dos valores dos desvios.

\subsection{Gráfico de Espalhamento de Moran}

A partir da plotagem dos valores de $\mathrm{Wz}$ em $\mathrm{Z}$ é possível traçar uma reta de regressão linear para tais valores cuja inclinação é equivalente ao Índice Global de Moran, NEVES et al (2000). Este Gráfico é chamado de Gráfico de Espalhamento de Moran (ANSELIN, 1996) e permite classificar os elementos de acordo com os valores de $\mathrm{Z}$ e Wz em quatro diferentes quadrantes Figura 1.

Os elementos situados no quadrante 1 , alto-alto (aa) apresentam valores altos de $\mathrm{Z}$ e altos também de Wz. Trata-se de um município com alto IDHM cercado por municípios de valores também altos. Os elementos situados no quadrante 2, baixo-baixo (bb) apresentam valores baixos de $\mathrm{Z}$ e baixos também de Wz. Trata-se de um município de baixo IDHM cercado por municípios com valores também baixos. Nos quadrantes Q1 e Q2 estão situados os clusters. Os elementos situados no quadrante 3 , alto-baixo (ab) apresentam valores altos de $\mathrm{Z}$ e baixos de $\mathrm{Wz}$, trata-se então de um município de alto
IDHM cercado por municípios com baixo IDHM. Finalmente, os elementos situados no quadrante 4 baixoalto (ba) apresentam valores baixos de $\mathrm{Z}$ e altos de $\mathrm{Wz}$, trata-se então de um município de IDHM baixo cercado por municípios de alto IDHM. Nos quadrantes Q3 e Q4 estão situados os outliers.

Neste trabalho foram construídos mapas que mostram tais elementos de acordo com a sua posição no gráfico de espalhamento de Moran bem como mapas que mostram o seu grau de significância visando facilitar o entendimento de tais relações espaciais.

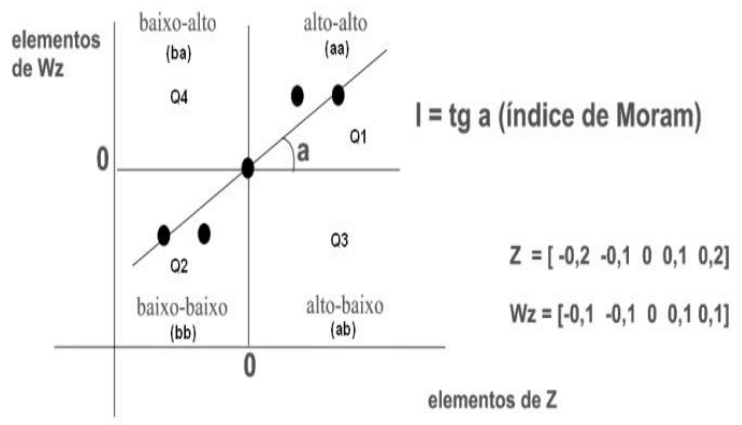

Fig.1- Gráfico de dispersão de Moran. Fonte: adaptado de NEVES et al (2000).

\section{METODOLOGIA}

O IDHM é um número que varia entre 0 e 1 . Quanto mais próximo de 1 , maior o desenvolvimento humano de um município. Pode ser calculado a partir da média geométrica de suas três componentes: IDHM Educação, IDHM Longevidade, IDHM Renda, todos obtidos a partir dos dados dos Censos Demográficos do IBGE. Todas as componentes possuem o mesmo peso.

As componentes são obtidas da seguinte forma:

O IDHM Longevidade mostra o número médio de anos que uma pessoa nascida em determinado município viveria a partir do nascimento, mantidos os mesmos padrões de mortalidade e é medido pela expectativa de vida ao nascer, calculada por método indireto.

O IDHM Educação é obtido pela média geométrica de dois indicadores: a escolaridade da população adulta que tem peso 1 e pelo fluxo escolar da população jovem que tem peso 2 . O primeiro é medido pelo percentual de pessoas de 18 anos ou mais de idade com ensino fundamental completo. O segundo é medido pela média aritmética do percentual de crianças de 5 a 6 anos frequentando a escola; do percentual de jovens de 11 a 13 anos frequentando os anos finais do ensino fundamental; do percentual de jovens de 15 a 17 anos com ensino fundamental completo e do percentual de jovens de 18 a 20 anos com ensino médio completo.

O IDHM Renda é obtido pela soma da renda de todos os residentes, dividida pelo número de pessoas que moram no município, ou seja, a renda municipal per capita. 


\subsection{Programas Utilizados}

Todo o trabalho foi realizado utilizando softwares livres, a estatística descritiva e o teste de normalidade foram realizados no $\mathrm{R}$, as operações de geoprocessamento para preparar os arquivos bem como a elaboração dos mapas foi realizada no QGIS enquanto que a Análise Exploratória Espacial dos dados foi realizada no Geoda.

O Ambiente R ( $\mathrm{R}$ Core Team, 2014) ou simplesmente $\mathrm{R}$ é uma linguagem de programação orientada a objetos criada em 1996 por Ross Ihaka e Robert Gentleman. Apresenta código fonte aberto, o que significa que pode ser modificado ou implementado com novos procedimentos desenvolvidos por qualquer usuário a qualquer momento. Possibilita a realização de operações matemáticas, a manipulação de vetores e matrizes, bem como a análise e manipulação de dados, com testes paramétricos e não paramétricos, modelagem linear e não linear, análise de séries temporais, análise de sobrevivência, simulação e estatística espacial, entre outros, além de apresentar facilidade na elaboração de diversos tipos de gráficos, com pleno controle do usuário sobre os gráficos criados. Com isso, consolida-se como uma importante ferramenta estatística e uma alternativa aos programas comerciais.

O QGIS (QGIS Development Team, 2014) é um software SIG com uma interface gráfica simples e atraente, escrito em C++ e Python e baseado nas bibliotecas Qt4. Suporta numerosos formatos vetoriais, raster, e bases de dados, e fornece uma ampla gama de funções de geoprocessamento raster e vetorial. Trata-se de uma aplicação multiplataforma que funciona em todas as principais versões do Unix, GNU/Linux, bem como Mac OsX e MS Windows. O projeto QGIS é o resultado do trabalho voluntário de um grupo de desenvolvedores, tradutores, autores de documentação e pessoas que ajudam no processo de lançamento de novas versões, identificando e divulgando as falhas do programa. É livremente distribuído com a licença GPL (GNU General Public License) e é um projeto oficial da Open Source Geospatial Foundation (OSGeo) (MANGHI, G.; CAVALINHENSE P. e NEVES V., 2011). Atualmente encontra-se na versão 2.12 .

Finalmente o GeoDA (ANSELIN et al, 2006) tratase de um programa livre criado pelo Centro de Análise Geoespacial e Computação da Universidade do Arizona que trabalha com Análise Exploratória de Dados, Análise Exploratória de Dados Espaciais e Regressão Espacial, uma das vantagens é que este programa trabalha com o formato shapefile, facilitando o compartilhamento dos resultado com os Sistemas de Informações Geográficas.

\subsection{Dados Utilizados}

Os dados referentes ao IDHM foram obtidos por meio da página da internet do Atlas do Desenvolvimento Humano no Brasil 2013 (Atlas Brasil) que disponibiliza uma tabela com as informações dos municípios que fazem parte da Região Semiárida Brasileira.
$\mathrm{O}$ arquivo vetorial com as informações dos 1135 municípios na escala 1:250.000 foram obtidos por meio da página da internet do Sistema de gestão da Informação e do Conhecimento do Semiárido Brasileiro (SIGSAB) de responsabilidade do Instituto Nacional do Semiárido.

\subsection{Procedimentos Metodológicos}

Os procedimentos realizados para alcançar os objetivos propostos estão descritos no fluxograma mostrado na Figura 2 a seguir.

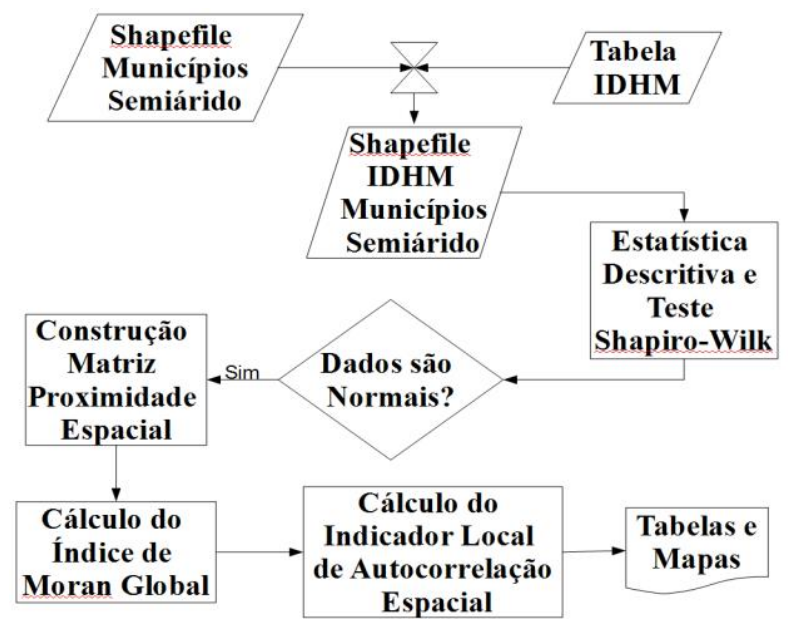

Figura 2 - Fluxograma dos procedimentos desenvolvidos no trabalho.

O primeiro passo foi realizar a ligação entre a tabela com as informações do IDHM e a tabela de atributos do arquivo vetorial, a partir de um tributo comum nas duas tabelas. Como resultado foi obtido o arquivo vetorial com as informações do IDHM para os 1135 municípios.

Os dados tabelados foram analisados para obter a estatística descritiva e submetidos ao teste de Normalidade de Shapiro-Wilk, pois a existência de normalidade é requisito para a realização das próximas etapas.

Após a verificação da normalidade construiu-se a matriz de proximidade espacial, na qual foi definida a estratégia de construção com base na contiguidade e considerando que os vizinhos teriam pesos iguais.

O próximo passo foi realizar o cálculo do Índice de Moran Global.

Após a confirmação da existência da autocorrelação espacial foi possível calcular os Indicadores Locais de Associação Espacial (LISA). A partir destes indicadores foram produzidos o Mapa de clusters e o Mapa de Significância dos clusters para a variável em análise.

\section{RESULTADOS E DISCUSSÃO}

A estatística descritiva dos dados pode ser observada na Tabela 1. Esta mostra as principais medidas de posição e dispersão referentes aos 1135 municípios analisados. A análise do coeficiente de variação $(\mathrm{CV})$ indica uma baixa variabilidade dos mesmos, sendo que todos os índices apresentaram CV abaixo de 7\% com exceção do IDHM 
Educação que apresentou CV de 12,63\% o que ainda assim, pode ser considerado baixo. Isto aponta para a existência de certa uniformidade do referido índice na região em estudo.

Tabela 1 - Estatística Descritiva.

\begin{tabular}{crrrr}
\hline & IDHM & $\begin{array}{c}\text { IDHM } \\
\text { Renda }\end{array}$ & $\begin{array}{r}\text { IDHM } \\
\text { Educacão }\end{array}$ & $\begin{array}{c}\text { IDHM } \\
\text { Longevidade }\end{array}$ \\
\hline $\mathbf{N}^{\mathbf{0}}$ obs. & 1135 & 1135 & 1135 & 1135 \\
Mínimo & 0,484 & 0,455 & 0,316 & 0,672 \\
Máximo & 0,720 & 0,710 & 0,714 & 0,846 \\
Média & 0,592 & 0,563 & 0,488 & 0,757 \\
Mediana & 0,591 & 0,561 & 0,485 & 0,760 \\
Desvio & 0,038 & 0,038 & 0,062 & 0,029 \\
Padrão & & & & \\
CV (\%) & 6,481 & 6,667 & 12,634 & 3,896 \\
\hline
\end{tabular}

A análise da média do IDHM permite afirmar que com exceção do IDHM Longevidade a situação das demais componentes e do IDHM geral situa a região semiárida como uma região de Baixo desenvolvimento humano; no que se refere à componente Educação, a situação é ainda mais preocupante, pois a classificação é Muito Baixo de acordo com a classificação do Atlas de Desenvolvimento Humano no Brasil mostrada na Figura 3.

No que se refere ao IDHM propriamente dito, na região semiárida $59,5 \%$ dos municípios são considerados como de baixo desenvolvimento humano, $39,2 \%$ são considerados como de médio desenvolvimento humano e apenas $0,7 \%$ são considerados como de alto desenvolvimento humano.

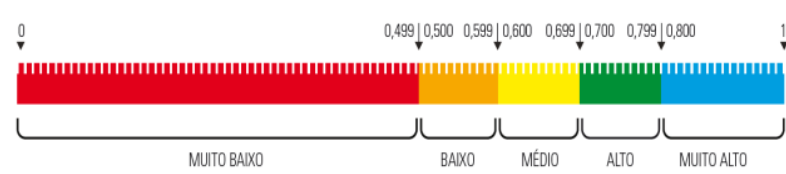

Figura 3-Faixas de classificação do IDHM. Fonte: Atlas de Desenvolvimento Humano no Brasil (PNUD, 2013).

A segunda etapa da análise consistiu em realizar o teste de normalidade para o índice em estudo. Na Tabela 2 pode-se observar o resultado do Teste de Shapiro-Wilk. Nela é possível afirmar que o índice em análise apresenta distribuição normal, ou seja, o requisito para realização da análise da autocorrelação espacial global foi atendido.

Tabela 2 - Teste de Shapiro-Wilk.

\begin{tabular}{ccc}
\hline Variáveis & W & p-valor \\
\hline IDHM & 0,9941 & 0,00018 \\
\hline
\end{tabular}

As Figuras 4 e 5 permitem comparar a distribuição espacial do IDHM 2010 classificada segundo as faixas de classificação do PNUD e em termos de desvios padrão da média.
A primeira classificação é adequada para a realidade do Brasil como um todo e quando aplicada apenas na região semiárida não consegue explicitar as diferentes realidades dos municípios. Por exemplo, na Figura 4 é possível perceber que a maioria dos municípios apresentam IDHM entre baixo e médio, e apenas 8 municípios apresentam IDHM Alto.

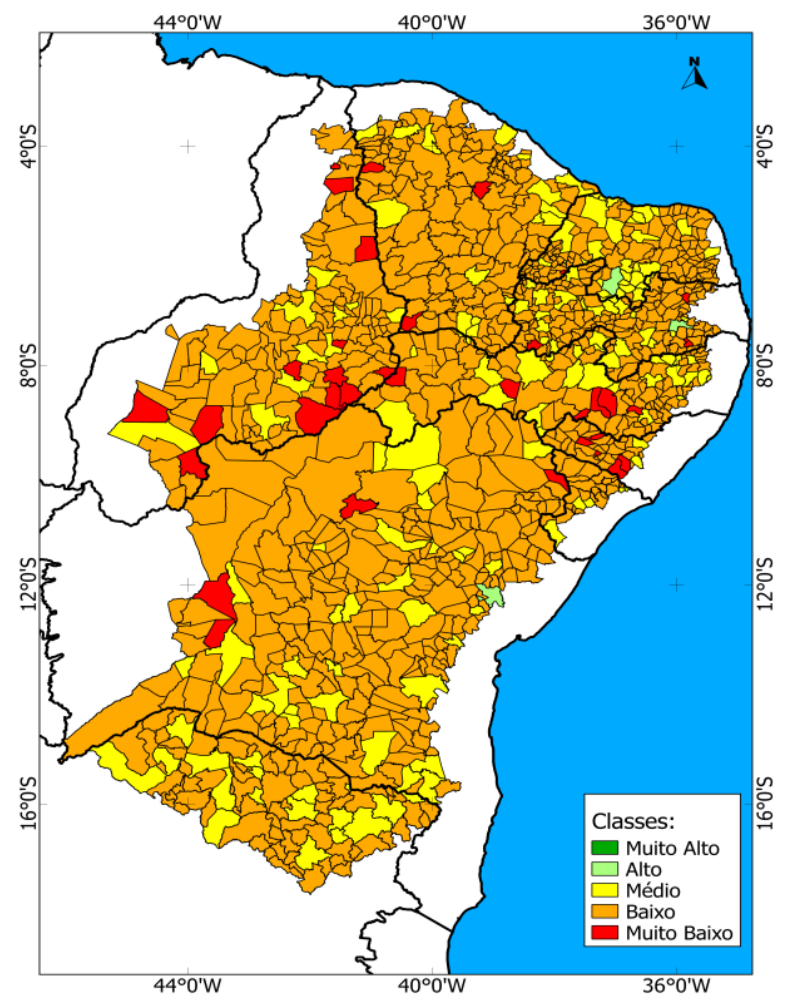

Figura 4- Distribuição espacial do IDHM 2010 na região semiárida brasileira, classificada a) de acordo com a classificação do PNUD.

A segunda classificação, baseada nos desvios padrão em torno da média dá uma maior clareza a respeito da situação a que cada município está submetido, evidenciando as relações entre os vizinhos, sendo por isso mais adequada aos objetivos deste trabalho. Na Figura 5 é possível verificar o quão heterogênea é a distribuição espacial do referido índice. Percebe-se claramente os municípios que se destacam (> 3 desvios padrão) em cada um dos estados em relação ao IDHM, como também as regiões nas quais o IDHM é mais baixo (<-2 desvios padrão), sendo que elas não necessariamente estão separadas, algumas vezes são vizinhas. 


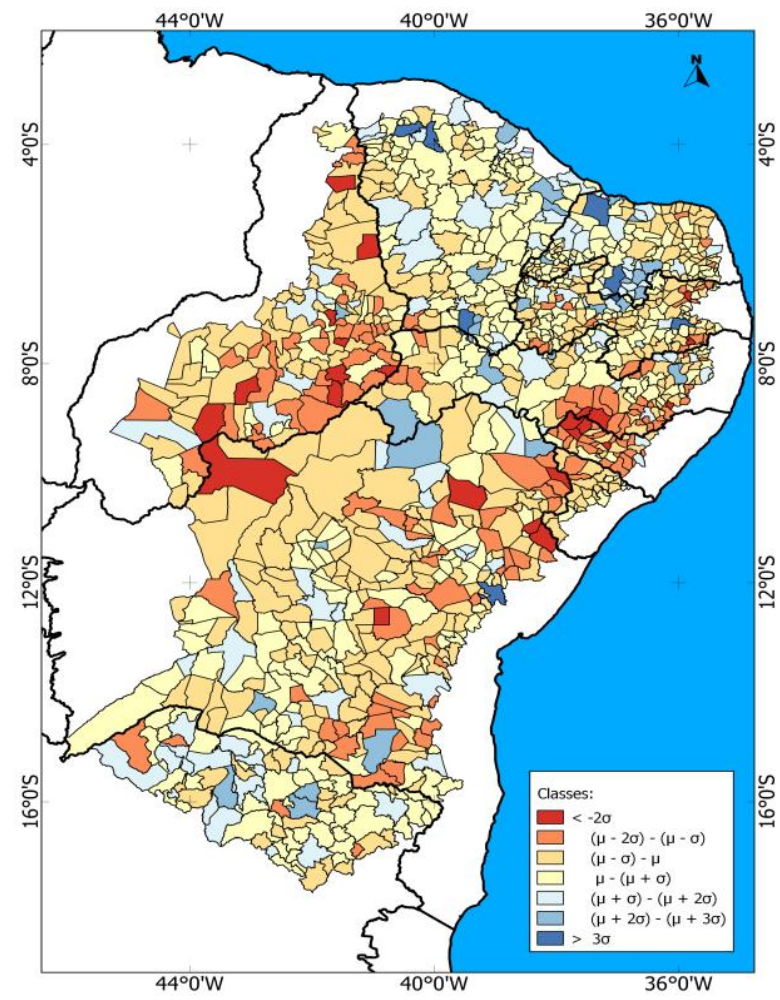

Figura 5- Distribuição espacial do IDHM 2010 na região semiárida brasileira, classificada de acordo com os desvios padrão em torno da média.

A Tabela 3 exibe os resultados do índice de Moran Global e o seu teste de significância. Para a variável sob análise o teste se mostrou significativo. Isto permite afirmar que existe a autocorrelação espacial no IDHM, ou seja, existem municípios nos quais a vizinhança é fator que influencia a determinação do referido índice.

Tabela 03 - Índice de Moran e Teste de Significância.

\begin{tabular}{ccc}
\hline Variáveis & Índice de Moran I & p-valor \\
\hline IDHM 2010 & 0,3507 & 0,05 \\
\hline
\end{tabular}

A Figura 6 mostra o gráfico de dispersão de Moran para o IDHM. Nele o Índice de Moran refere-se à inclinação da reta. O Gráfico é dividido em quadrantes, sendo que o quadrante superior direito refere-se aos clusters de alto valor, ou seja, um município com alto IDHM é vizinho de municípios também com alto IDHM; o quadrante inferior esquerdo refere-se aos clusters de baixo valor, ou seja, um município com baixo IDHM é vizinho de municípios com baixo IDHM; além disso, mostram municípios cujo IDHM é de baixo valor enquanto seus vizinhos são de alto valor sendo denominados de outliers, os quais estão localizados no quadrante superior esquerdo; e os outliers de alto valor, ou seja, municípios com alto valor e vizinhança de baixo valor localizado no quadrante inferior direito.

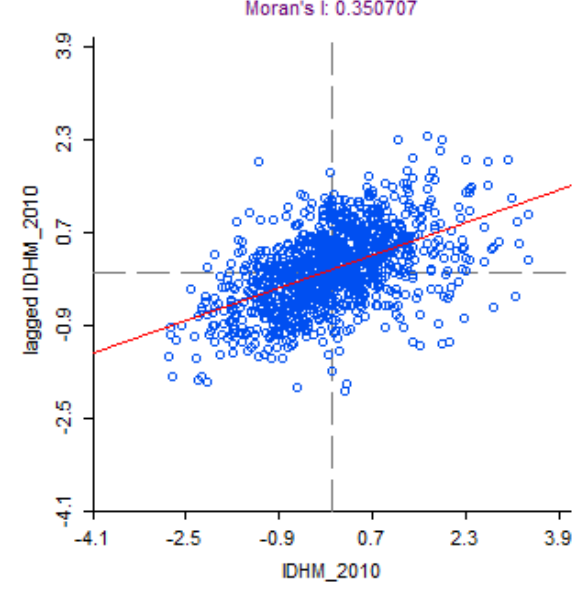

Figura 6 - Gráficos de dispersão de Moran para o IDHM 2010 da Região semiárida Brasileira.

A Figura 8 apresenta o resultado do Índice de Moran Local ou LISA para o IDHM 2010. Nela é possível identificar os clusters e outliers espaciais. A formação dos clusters está associada à existência de municípios de classificação >3 desvios padrão da média (cluster de valor alto) e <-2 desvios padrão da média (cluster de valor baixo) na Figura 5. Percebe-se que os clusters de Alto valor estão localizados principalmente no estado do Ceará, Rio Grande do Norte, e Minas Gerais; enquanto que os clusters de baixo valor estão distribuídos nos estados da Bahia, Piauí, Pernambuco, com destaque para Alagoas e Sergipe nos quais boas partes dos municípios localizados na sua região semiárida fazem parte do referido cluster.

A existência de clusters de altos valores pode significar a existência de políticas públicas que venham sendo implantadas com sucesso não apenas em um município, mas também nos seus vizinhos o que é algo desejável e que deve ser estimulado. Conhecer os fatores que levam à formação destes clusters pode ser útil para que estas experiências possam ser reproduzidas em outras regiões.

Seguindo o mesmo raciocínio, os municípios que apresentam clusters de baixos valores podem indicar regiões prioritárias para que sejam implantado políticas públicas que busquem mudar a realidade destas regiões, como também conhecer os fatores que levam a que um conjunto de municípios tenha índices de desenvolvimento mais baixos que os seus vizinhos.

O oposto destas situações são os outliers, municípios que são como "ilhas", sendo que um outlier baixo-alto significa os municípios do seu entorno apresentam um alto índice enquanto que ele apresenta um índice menor. Já o outlier alto-baixo apresenta vizinhança com baixos valores em relação ao seu valor do índice. 


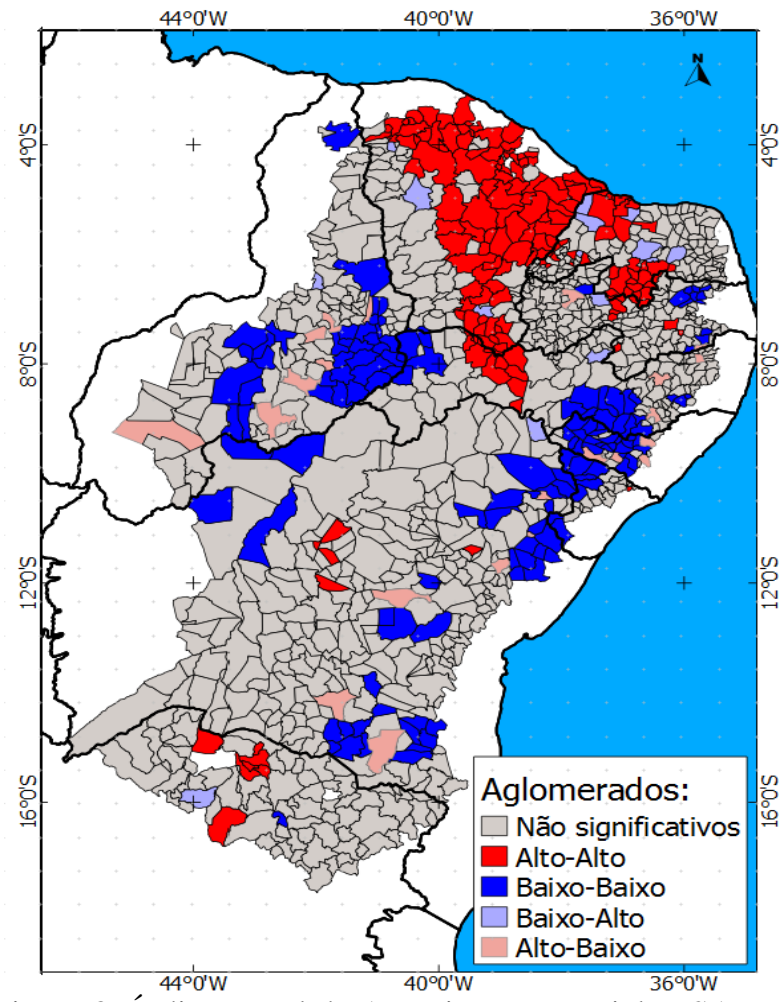

Figura 8- Índice Local de Associação Espacial-LISA para o IDHM da região Semiárida Brasileira.

\section{CONCLUSÕES}

Este trabalho teve como objetivo analisar a existência de algum padrão de distribuição espacial para os dados do IDHM nos 1135 municípios que compõe a região semiárida Brasileira.

A partir da análise do índice de Moran Global e do Índice Local de Autocorrelação Espacial-LISA foi possível identificar a existência de autocorrelação espacial no IDHM 2010 e a análise da autocorrelação local revelou a existência de clusters de altos e de baixos valores, em todos os estados estudados.

Os clusters de altos valores se concentraram no norte e no sul da região semiárida enquanto que os de baixos valores se concentraram no centro da região.

Sugere-se a continuidade dos trabalhos para investigar quais são os fatores que levam à formação destes clusters, e no caso dos clusters de altos valores tentar reproduzi-los em outras regiões. Com relação aos clusters de baixos valores estes podem indicar regiões prioritárias para ação por parte dos governos municipais e estaduais com o objetivo de reduzir o baixo desempenho do IDHM na região Semiárida.

\section{REFERÊNCIAS BIBLIOGRÁFICAS}

ANSELIN, L. Local Indicators of Spacial Association LISA. Geographical Analysis. v.27, n.2, p.93-115, 1995.

ANSELIN, L. The Moran scatterplot as ESDA tool to assess local instability in spatial association. In: Fisher, M.; Scholten, H. J.; Unwin, D. Spatial
Analytical Perspectives on GIS. London: Taylor \& Francis, 1996, p. 111-126.

ANSELIN, L.; IBNU S.; YOUNGIHN K. GeoDa: An Introduction to Spatial Data Analysis. Geographical Analysis 38 (1), 5-22, 2006.

GONCALVES, E. O padrão espacial da atividade inovadora Brasileira: uma análise exploratória. Estud. Econ., São Paulo, v. 37, n. 2, June 2007. Disponível em: http://www.scielo.br/scielo.php?pid=S010141612007000200007\&script=sci_arttext. Acessado em 10 de abril de 2013.

INSA. Sinopse do Censo Demográfico para o Semiárido Brasileiro. Campina Grande: INSA, 2012.103p.

MANGHI, G.; CAVALINHENSE P.; NEVES V. Quantum GIS: Um desktop potente e amigável. Revista FOSSGIS Brasil, Junho 2011.www.fossgisbrasil.com.br. Acesso em: 01/04/2013.

NEVES, M. C.; LUIZ, A. J. B. Distribuição espacial da cultura de café no Estado de São Paulo. Embrapa Meio Ambiente. Boletim de Pesquisa e Desenvolvimento, 2006.

NEVES, M. C. et al. Análise exploratória espacial de dados sócio-econômicos de São Paulo. Anais do GIS Brasil 2000, 2000.

QGIS DEVELOPMENT TEAM, 2014. QGIS Geographic Information System. Open Source Geospatial Foundation Project. Disponível em: http://qgis.osgeo.org. Acesso em: 20 de maio de 2014.

PEROBELLI, F. S.; FERREIRA, P. G.; FARIA, W. R. Análise de convergência espacial do pib per-capita em minas gerais: 1975-2003. In: XI Encontro Regional de Economia, 2006, Fortaleza. Nordeste: Estratégias de Desenvolvimento Regional. Fortaleza: BNB, 2006.

PNUD. Índice de Desenvolvimento Humano Municipal Brasileiro. - Brasília: PNUD, Ipea, FJP, 2013.96 p.

R Core Team (2014). R: A language and environment for statistical computing. R Foundation for Statistical Computing, Vienna, Austria. URL http://www.Rproject.org/. Acesso em: 04 de maio de 2014. 